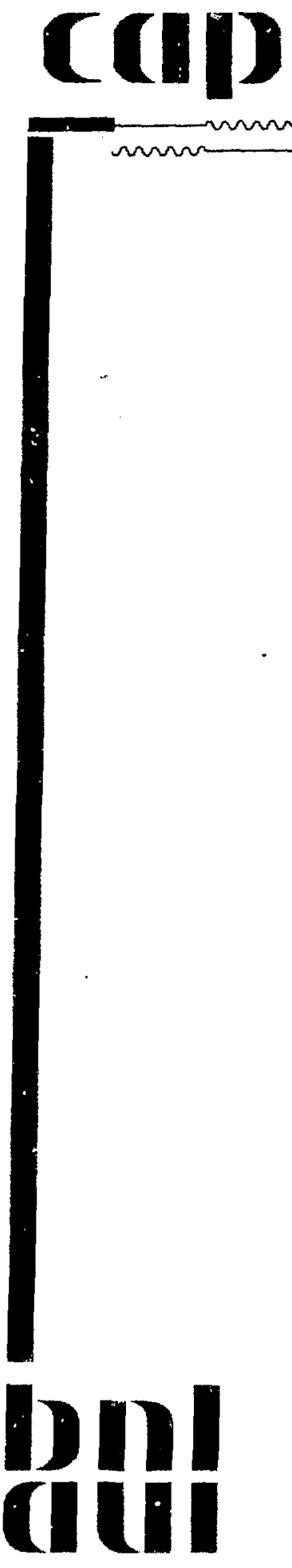

\title{
Quadrupole Magnets Measurement
}

Xijie Wang

Center for Advanced Accelerator Physics

Department of Physics, UCLA

and

Cosmore Sylvester

National Synchroton Light Source

Brookhaven National Laboratory

\section{CENTER FOR ACCELERATOR PHYSICS}

\author{
BROOKHAVEN NATIONAL LABORATORY \\ ASSOCIATED UNIVERSITIES, INC. \\ Under Contract No. DE-ACO2-76CHDOO16 with the \\ UNITED STATES DEPARTMENT OF ENERGY
}




\section{DISCLAIMER}

This report was prepared as an account of work sponsored by an agency of the United States Government. Neither the United States Government nor any agency thereof, nor any of their employees, nor any of their contractors, subcontractora, or their employees, makes any warranty, express or implied, or assume, any legal liability or responsibility for the accuracy, completeness, or usefulness of any information, apparatus, product, or process disclosed, or represents that its use would not infringe privately owned rights. Reference herein to any specific commercial product, process, or service by trade name, trademark, manufacturer, or otherwise, does not necessarily constitute or imply its endorsement, recommendation, or favoring by the United States Government or any agency, contractor or subcontractor thereof. The views and -opinions of authors expressed herein do not necessarily state or reflect thuse of the United States Government or any agency, contractor or subcontractor thereof. 


\title{
QUADRUPOLE MAGNETS MEASUREMENT
}

\author{
Xijie Wang \\ Center For Advanced Accelerators Physics \\ Department of Physics, UCLA \\ and \\ Cosmore Sylvester \\ National Synchrotron Light Source \\ Brookhaven National Lab
}

\begin{abstract}
A rotating coil setup is designed for quadrupole magnet measurement at the Accelerator Test Facility (ATF); Hall probe measurement was also performed for one of each type of quadrupole magnet. Both mechanical and magnetic properties of the quadrupole magnets were measured, the results are reported here.
\end{abstract}

\section{INTRODUCTION}

The Brookhaven Accelerator Test Facility (ATF) is a dedicated facility for the Free-electron Laser and other Accelerator Physics experiments. A low-emittance and high-brightness electron beam is required for these experiments. One of the main obstacles to preserving the high quality electron beam produced by the photocathode RF gun is the magnetic transport line[1], so a program was initiated at the ATF to measure the quadrupole magnets.

The quadrupole magnets used in the ATF post-linac magnetic transport line come from two sources. Fourteen new quadrupole magnets are manufactured to ATF specifications by IHEP of China Academia Sinica; and twenty one quadrupole magnets are spare SLAC quadrupoles. 
The laser linac beam line[1] and FEL line[2] demand detailed knowledge of each magnet, such as the integrated gradient as a function of the excitation current, harmonic components, and the displacement between mechanical and magnetic centers. A long flat coil with one side passing through the magnet center and a Hall probe were adopted for our measurement.

\section{QUADRUPOLE MAGNETIC MEASUREMENT.}

\section{Rotating Coil Fixture.}

The main advantage of the long fiat coil is the appearance of all harmonic signals and its simplicity. The rotating coil fixture is illustrated in Figure 1. Both ends of the fixture are attached to the quadrupole being measured. Each end of the fixture is supported by an $\mathrm{X}-\mathrm{Y}$ translator stage so the transverse position of the coil can be adjusted. To accommodate both long and short quadrupoles, the longitudinal position of the coil can also be changed. The radius of the Chinese quadrupole and the SLAC quadrupole are $2.25 \mathrm{~cm}$ and $1.52 \mathrm{~cm}$, respectively, so the radius of the G-10 Iotating rod was chosen to be $1.27 \mathrm{~cm}$. To increase the signal level, a multi-turn coil ( $4 \times 12$ turns with the effective area $1.2 \mathrm{~mm} \times 3.6$ $m m$ ) was used. The coil board was manufactured by MULTIWIRE CORP. to our specifications. The wire was placed in " $B$ " stage epoxy on a substrate using ultrasonic technology (Fig.2). The coil board was clamped on the G-10 rod by precision pins (Fig.3). For the convenience of distinguishing external noise, a variable speed motor $(0-125 R P M)$ was employed to drive the G-10 rod. 


\section{Mechanical Center of the Quadrupole Magnet.}

Before mounting the quadrupole magnets for harmonic measurement, each magnet was inspected. The aperture and individual pole of each magnet was measured. For Chinese quadrupoles, the radial placement error of the pole is less than $38 \mu m$. Large errors $(75-375 \mu m$ ) were found in most SLAC quadrupoles however. The error was caused by a combination of improper assembly and deformation of the magnets; a mandrel was made for assembling the SLAC quadrupole magnets.

To measure the displacements between the mechanical and magnetic centers, the mechanical center of the quadrupole should first be located accurately. An optical survey was first used to place the G-10 rod center in the mechanical center of the quadrupole magnet. The method is time-consuming and inaccurate for our magnets because the length of the quadrupole is relatively short. Since our objective is to place the rod and the magnet in the same mechanical center, precise survey of the quadrupole magnet is not essential. To achieve the above goal, a dial indicator was clamped on the rod at each end of the quadrupole magnet. As the rod was rotated manually, we obtained the reading for all four poles at each end of the quadrupole; the reading can be made as close as possible by moving the translator stages. In this way, we were be able to locate the mechanical center within to $\pm 12.7 \mu m$.

\section{Harmonic Measurement.}

All quadrupole magnets are powered by a $10 \mathrm{~V} / 70 \mathrm{~A}$ Bruker Power Supply with a $\pm 0.01 \%$ stability. The signal was analyzed by an HP Dynamic Signal 
Analyzer though a BNC coaxial cable with a floating ground. To achieve the largest signal level, the excitation currents were set to the maximum specification for both Chinese and SLAC quadrupoles(31.5 and 7.0 A, respectively).

Various rotation speeds of the motor were tested for identification of the true harmonic signal and noise level. It was found that the highest speed should be used for the best signal-to-noise ratio. The main problem with fast rotation is the vibration associated with it and a large sideband around the quadrupole signal.

Fig.4 illustrates a typical time domain signal induced by the rotation coil in the quadrupole field. The power spectrum of the residual field of the quadrupole is plotted in Fig.5. Figures 6 and 7 are power spectra of the quadrupole at the mechanical and magnetic centers, respectiveiy. Fig. 8 is the rms voltage of the signal for each harmonic component.

The voltage induced on the coil is proportional to the integrated magnetic field. The difficulty in determining the absolute magnetic field very accurately for ou: situation comes from the following: the dimension of the multi-turn wire coil is not well defined, and the impedance is mismatched between the coaxial cable and the coil. Table 7 gives the voltages induced on the quadrupole magnets due to the quadrupole component. When combined with the Hall probe measurement for one of each type of quadrupole magnet, the excitation curve for each quadrupole magnet can be obtained.

Hall Probe Measurement.

The F.W. BELL Hall probe model 8860 with probe HTR - 0608 was used in our measurements. The output of the Hall probe was measured by an HP 3456 
Digital Voltage Meter to achieve more significant digits. Since the displacements of the magnetic centers to the mechanical centers have been obtained in the harmonic measurement, the position of the Hall probe in the transverse plane is not critical as long as the Hall probe is parallel to the magnetic poles. We have calibrated all translator stages used in Hall probe measurement by a dial indicator. The accuracy is found to be good within a few micrometers.

The longitudinal profiles of the magnets were measured at different horizontal locations (Fig. $9-12$ ) to obtain the effective lengths of the quadrupoles. The effective length of a quadrupole is given by numerical integration of the fields along the longitudinal direction.

To obtain the excitation curve, the magnets were first excited to the full power, then the current were brought to zero. Currents were subsequently gradually increased to the specified values. A horizontal scan of the magnetic field was performed for each current setting, the difference of the magnetic field and horizontal position were used to calculate the field gradient.

\section{DISCUSSION AND ANALYSIS.}

The effect of the displacement of the rotating coil from the magnetic center in a multipole magnet is predominated by the lower order harmonics. For a quadrupole magnet, it is the dipole signal. To locate the magnetic center of a quadrupole, the X-Y translator stages were used to eliminate the dipole signal. The accuracy of these translator stages is $\pm 5 \mu \mathrm{m}$. To study how the mechanical vibrations in the measuriag coil affect the location of the magnetic center and 
harmonic signal, we repeatedly measured a Chinese quadrupole. Table 1 lists the displacement between magnetic and mechanical centers and harmonic signals for each measurement. From this table we determined that the accuracy of locating the magnetic center is roughly $\pm 10 \mu m$.

It is also possible to determine the displacement between the mechanical and magnetic centers by the ratio of the dipole signal voltage to the quadrupole signal voltage times the width of the coil. Assume both dipole and quadrupole component have the same effective length $L, \delta$ is the displacement between the two centers, $W$ is the coil width and $\delta \ll W$. By the constant of the gradient of the quadrupole magnet, we have:

$$
\delta=\frac{B_{1} L}{B_{2} L} W=\frac{V_{1}}{V_{2}} W
$$

where $B_{1}$ is dipole magnetic field, and $B_{2}$ is the magnetic field due to the quadrupole component at the radius $W ; V_{1}$ and $V_{2}$ are voltages due to the dipole and quadrupole magnetic field components. Table 2 compares the measured displacement using the translator stage with the calculated value. The agreement is within the accuracy of our system.

For a symmetrical multipole magnet, the magnetic field can be expressed in a power series in cylindrical coordinates,

$$
B=\sum{ }_{2} C_{N, m} r^{m-1} e^{-1\left(m \theta+\alpha_{N, m}\right)}
$$

where $2 N$ is the pole number, and $m=N(2 k+1), k=0, \cdots, \infty$. 
For a symmetrical quadrupole, the lowest allowable harmonic is the dodecapole. In our harmonic measurements, both sextuple and octopole were observed. The octopole can be caused by radial asymmetry and sextupole by one pole's angular displacement[4]. Mechanical measurements confirmed that these asymmetries do exist, but the magnitude of the sextupole is larger than expected. The possibility of an external source causing the sextupole was checked by changing the rotation frequency of the motor. The effects of wiring is negligible for an iron dominant magnet. We also investigated the effect of wire distribution in a multi-turn coil, the radial variation of the wire has been studied[5], which can be treated as a single turn coil with equivalent current and radius. Let $\Delta$ be the angular difference between neighboring wires; the four-turn coil can be treated as four different multipole fields with different phases, so we have the following:

$$
B=\sum \imath C_{N, m} r^{m-1} e^{-\imath\left(m \theta+\alpha_{N, m}\right)} \times \sum_{j=0}^{3} \exp (\imath j m \Delta) .
$$

The above expression can be reduced to,

$$
B=4 \sum{ }_{2} C_{N, m} \cos \frac{m \Delta}{2} \cos (m \Delta) r^{m-1} e^{-\mathrm{r}\left(m \theta-\frac{3}{2} m \Delta \div \alpha_{N, m}\right)}
$$

As $\Delta$ approaches zero, the field equals the sum of the four multipole fields. It can be seen that the angular distribution of the wire will affect both the amplitude and phase of the signal, but can not be the source of the sextupole. That leaves only the simulation of the sextupole by the rotating coil. Inspecting the G-10 rod, mechanical relaxation was observed. We believe that the sextupole was the combined effect of the asymmetry of the quadrupole magnet, the sideband of the 
quadrupole signel and the simulation of the harmonic by the coil. This effect on the post linac electron beam is negligible.

The Hall probe and the readout from the HP DVM were calibrated by an NMR from $1 K G$ to $10 K G$. The linearity of the probe was assumed for the fields out of the calibration range. The background magnetic field was observed in the range from 0.2 to 0.5 Gauss. When the average function of the DVM was used, 0.1 Gauss repeatability was achieved.

The main sources of error for the Hall probe measurement are the finite dimension of the crystal used in the probe and the survey error. These effects are especially important in the low field region, i.e., near the magnetic centers, the proper choices of the horizontal offsets for effective length measurement can reduce the contribution from those effects. The effective length of the quadrupole is defined as,

$$
L=\frac{1}{G_{0}} \int G d \ell
$$

where $G=d B_{y} / d x, G_{0}$ is the field gradient in the central region of the quadrupole. Generally speaking, $L$ is not independent of the position. For a small offset from the magnetic center, it can be treated as constant.

To study the effect of the survey error on the longitudinal profile and magnetic field gradjent of the quadrupole magnet, we ignore the edge effect of the quadrupole. Since the Hall probe is positioned in such a way that only $B_{y}$ will contribute to the signal, the survey error in the vertical dimension can be neglected for a longitudinal field scan. Let the horizontal offset of the longitudinal scan trace at the center of the quadrupole be $x_{0}$. Then the maximum horizon- 
tal position error is equal to half of the survey error; that is, $\Delta x=e / 2$. The longitudinal field profile is:

$$
B=G\left(x_{0} \pm \Delta x\right)=B_{0}\left(1 \pm \frac{e}{2 x_{0}} \frac{2 z}{L}\right)
$$

where $z$ is the longitudinal displacement measured from the center of the quadrupole, $L$ is the quadrupole length, $B_{0}=G x_{0}$. The ratio of the the maximum error field to $B_{0}$ is:

$$
\frac{\Delta B}{B_{0}}=\frac{e}{2 x_{0}}
$$

This formula indicates that the fluctuation of the magnetic field in the central region of the quadrupole is determined by the ratio of the survey error to the reference offset.

The effect of the survey error on the field gradient can also be studied. The angle between the crystal and the horizontal plane can be expressed as $\phi=e / R$, where $e$ is the survey error and $R$ is the radius of the survey target. The field gradient is

$$
G=\frac{\Delta B_{y}}{\Delta x \cos (\phi)}=G_{0}\left(1+\phi^{2}\right)
$$

Since $\phi$ is on the order of $10^{-3}$, the survey error on the field gradient is negligible. It can be shown that the contribution from the vertical error displacement is second order as well.

Finally, the error of the transverse displacement measurement on the magnetic field gradient will be investigated in the following. Let $e$ be the displacement 
measurement error, and $\Delta x$ be the displacement. The field gradient is then

$$
G=\frac{\Delta B}{\Delta x \pm e}=G_{0}\left(1 \mp \frac{e}{\Delta x}\right)
$$

where $G_{0}=\Delta B / \Delta x$. The above formula shows that an accurate displacement measurement is very important for magnetic field gradient measurements since $\Delta x$ itself is typically a small number. Using the the above formulas, the error for integrate field gradient estimated to be about $0.5 \%$.

Combining table 7 and table $8-11$, the linear excitation function can be obtained for each quadrupole magnet. Table 12 lists the results.

\section{ACKNOWLEDGEMENTS}

The authors are grateful for the technical support provided by C. Biscardi and G. Stenby. We are also thankful for many helpful discussions with L. Solomon, H. Kirk and A.van Steenbergen. We also wish to acknowledge the editing contribution of Ty Foshe of UCLA.

\section{REFERENCES}

1. Xijie Wang and H.G. Kirk, BNL-43307.

2. A.van Steenbergen, "FEL OPTICS", Private Communication.

3. J.K. Cob and R. Cole, MT-1(1965) p.431.

4. J.K. Cob and J.J. Muray, SLAC-39.

5. K. Halbach, PEP-208. 


\section{List of Tables}

Table 1 Displacement and harmonic measurement for Chinese quadrupole type 2 no. 3.

Table 2 Comparison Between the measured and calculated displacement magnetic center relative to the mechanical center.

Table 3 Harmonic measurement for type 1 Chinese quadrupoles.

Table 4 Harmonic measurement for type 2 Chinese quadrupoles.

Table 5 Harmonic measurement for short SLAC quadrupoles.

Table 6 Harmonic measurement for short SLAC quadrupoles.

Table 7 The voltage induced by quadrupole component on the coil.

Table 8 The excitation table for Chinese quadrupole type 1 no. 4.

Table $\theta$ The excitation table for Chinese quadrupole type 2 no. 3.

Table 10 The excitation table for short SLAC quadrupole 153.

Table 11 The excitation table for long SLAC quadrupole 135.

Table 12 The list of linear excitation relation. 


\section{List o.: Figures}

Fig. 1 ATF rotating coil fixture.

Fig. 2 Multi-turn coil sketch.

Fig. 3 Coil board assembly sketch.

Fig. 4 Time varied voltage induced by the quadrupole.

Fig. 5 The power spectrum of the quadrupole before the magnet was powered.

Fig. 6 The power spectrum of the quadrupole at the mechanical center.

Fig. 7 The power spectrum of the quadrupole at the magnetic center.

Fig. 8 RMS voltage induced on the coil by each harmonic.

Fig. 9 Longitudinal profile of Chinese quad type I.

Fig. 10 Longitudinal profile of Chinese quad type II.

Fig. 11 Longitudinal profile of SLAC short quadrupole.

Fig. 12 Longitudinal profile of SLAC long quadrupole. 
Table 1 Displacement and harmonic mersurements for Chinese quadrupole type 2 No.3

\begin{tabular}{llllll}
\hline Test No. & $B_{3} / B_{2}$ & $B_{4} / B_{2}$ & $B_{6} / B_{2}$ & $B_{10} / B_{2}$ & $\delta(\mu m)$ \\
1. & 0.54 & 0.11 & 0.07 & 0.04 & 46 \\
2. & 1.19 & 0.12 & 0.03 & 0.05 & 35 \\
3. & 0.85 & 0.12 & 0.03 & 0.05 & 42 \\
4. & 0.65 & 0.11 & 0.03 & 0.04 & 52 \\
5. & 1.25 & 0.24 & 0.07 & 0.05 & 45 \\
6. & 0.57 & 0.10 & 0.04 & 0.06 & 32 \\
\hline
\end{tabular}

All harmonic ratio is in \%.

The average radius of the coil is about $11.0 \mathrm{~mm}$. 
Table 2 Comparison between the measured and calculated displacements magnetic center relative to the mechanical center

\begin{tabular}{llllll}
\hline Magnet Type & $\delta_{\text {mess. }}$ & $\hat{\boldsymbol{\delta}}_{\text {calc. }}$ & Magnet Type & $\delta_{\text {meas. }}$ & $\delta_{\text {calc. }}$ \\
CS 1 & 25 & 50 & CS 2 & $<10$ & 8.7 \\
CS 3 & $<10$ & 8.3 & CS 4 & 28.3 & 38.1 \\
CS 5 & 41.3 & 64.1 & CS 6 & 10 & 10.8 \\
CS 7 & 40 & 35.1 & CS 8 & 50 & 70 \\
CS 9 & 22.4 & 21.9 & CS 10 & 31.6 & 42.3 \\
CL 1 & 10 & 11.4 & CL 2 & 53.9 & 56.4 \\
CL 3 & 31.6 & 61.8 & CL 4 & 22.4 & 28.8 \\
SS 179 & 20 & 35.4 & SS 157 & 36.1 & 52.1 \\
SS 158 & 80.6 & 100 & SS 163 & 72.1 & 77.9 \\
SS 182 & 86 & 122 & SS 231 & 86 & 98.1 \\
SS 218 & 72.1 & 65.5 & SS 201 & 98.5 & 106.3 \\
SS 242 & 295.5 & 312.5 & SS 155 & 72.1 & 65.8 \\
SS 154 & 80.6 & 81.5 & SS 224 & 92.2 & 108.7 \\
SS 153 & 20 & 20.3 & SS 204 & 98.5 & 99.5 \\
SL 130 & 198.5 & 186.8 & SL 125 & 233.2 & 238.7 \\
SL 119 & 102.9 & 124.9 & SL 141 & 70.7 & 85.2 \\
SL 104 & 108.2 & 127.3 & SL 135 & 94.3 & 89 \\
\hline All & 20.3 &
\end{tabular}

All unit is in micrometer, the average width of the coil is $11 \mathrm{~mm}$.

CS - Chinese quads type 1 . CL - Chinese quads type 2.

SS - SLAC Short quads. SL - SLAC Long quads. 
Table 3 Harmonic measurements for type 1 Chinese quadrupoles

\begin{tabular}{llllll}
\hline Series No. & $B_{3} / B_{2}$ & $B_{4} / B_{2}$ & $B_{5} / B_{2}$ & $B_{6} / B_{2}$ & $B_{10} / B_{2}$ \\
\hline 1. & 0.34 & 0.15 & 0.08 & 0.05 & 0.06 \\
2. & 0.70 & 0.13 & 0.06 & 0.03 & 0.05 \\
3. & 0.68 & 0.04 & 0.04 & 0.05 & 0.06 \\
4. & 0.53 & 0.07 & 0.07 & 0.06 & 0.05 \\
5. & 0.93 & 0.15 & 0.04 & 0.04 & 0.04 \\
6. & 0.70 & 0.13 & 0.07 & 0.04 & 0.06 \\
7. & 1.27 & 0.35 & 0.09 & 0.10 & 0.03 \\
8. & 0.45 & 0.09 & 0.05 & 0.06 & 0.06 \\
9. & 0.50 & 0.16 & 0.04 & 0.05 & 0.05 \\
10 & 0.57 & 0.16 & 0.04 & 0.03 & 0.06 \\
\hline
\end{tabular}

${ }^{1}$ All harmonic component in \%.

2 The average radius of the coil is $11.0 \mathrm{~mm}$.

Table 4 Harmonic measurements for type 2 Chinese quadrupoles

\begin{tabular}{|c|c|c|c|c|c|}
\hline Series No. & $B_{3} / B_{2}$ & $B_{4} / B_{2}$ & $\bar{B}_{5} / B_{2}$ & $B_{6} / B_{2}$ & $B_{10} / B_{2}$ \\
\hline 1. & 0.86 & 0.06 & $0 \times 4$ & 0.06 & 0.06 \\
\hline 2. & 0.20 & 0.07 & 0.06 & 0.08 & 0.05 \\
\hline 3. & 0.57 & 0.10 & 0.03 & 0.04 & 0.06 \\
\hline 4. & & 0.16 & & 0.05 & 0.06 \\
\hline
\end{tabular}


Table 5 Harmonic measurements for short SLAC quadrupoles

\begin{tabular}{llllll}
\hline Series No. $B_{3} / B_{2}$ & $B_{4} / B_{2}$ & $B_{5} / B_{2}$ & $B_{6} / B_{2}$ & $B_{10} / B_{2}$ \\
\hline 153 & 0.46 & 0.36 & 0.08 & 0.12 & 0.03 \\
154 & 0.38 & 0.18 & 0.10 & 0.14 & 0.04 \\
155 & 0.11 & 0.16 & 0.10 & 0.14 & 0.08 \\
157 & 1.23 & 0.23 & 0.05 & 0.18 & 0.07 \\
158 & 0.43 & 0.23 & 0.09 & 0.07 & 0.06 \\
163 & 0.31 & 0.08 & 0.02 & 0.16 & 0.05 \\
179 & 0.82 & 0.25 & 0.06 & 0.14 & 0.03 \\
182 & 0.34 & 0.12 & 0.03 & 0.17 & 0.07 \\
201 & 0.71 & 0.18 & 0.10 & 0.12 & 0.05 \\
204 & 0.52 & 0.18 & 0.06 & 0.13 & 0.05 \\
218 & 0.39 & 0.07 & 0.04 & 0.15 & 0.06 \\
224 & 0.41 & 0.15 & 0.05 & 0.21 & 0.05 \\
231 & 0.29 & 0.14 & 0.06 & 0.20 & 0.06 \\
242 & 0.20 & 0.19 & 0.06 & 0.16 & 0.06 \\
\hline
\end{tabular}

Table 6 Harmonic measurements for long SLAC quadrupoles

\begin{tabular}{llllll}
\hline Series No. & $B_{3} / B_{2}$ & $B_{4} / B_{2}$ & $B_{5} / B_{2}$ & $B_{6} / B_{2}$ & $B_{10} / B_{2}$ \\
\hline 104 & 0.19 & 0.18 & 0.08 & 0.20 & 0.08 \\
119 & 0.19 & 0.16 & 0.05 & 0.19 & 0.04 \\
125 & 0.46 & 0.21 & 0.07 & 0.11 & 0.05 \\
130 & 1.15 & 0.29 & 0.12 & 0.17 & 0.06 \\
135 & 0.26 & 0.08 & 0.02 & 0.16 & 0.06 \\
141 & 0.22 & 0.20 & 0.03 & 0.17 & 0.04 \\
\hline
\end{tabular}


Table 7 The voltage induced by quadrupole component on the coil.

\begin{tabular}{llll}
\hline Mag. Type & $V_{r m s}(m V)$ & Mag. Type & $V_{r m o}(m V)$ \\
\hline CS 1 & 32.85 & CS 2 & 32.89 \\
CS 3 & 32.75 & CS 4 & 32.75 \\
CS 5 & 32.81 & CS 6 & 32.82 \\
CS 7 & 32.66 & CS 8 & 32.78 \\
CS 9 & 32.65 & CS 10 & 32.79 \\
\hline CL 1 & 64.92 & CL 2 & 64.66 \\
CL 3 & 64.87 & CL 4 & 64.76 \\
\hline SS 179 & 58.25 & SS 157 & 58.30 \\
SS 158 & 57.75 & SS 163 & 58.54 \\
SS 182 & 57.90 & SS 231 & 57.71 \\
SS 218 & 57.80 & SS 201 & 57.15 \\
SS 242 & 58.55 & SS 155 & 58.75 \\
SS 154 & 59.19 & SS 224 & 58.04 \\
SS 153 & 58.59 & SS 204 & 59.01 \\
\hline SL 104 & 113.53 & SL 119 & 115.05 \\
SL 125 & 115.18 & SL 130 & 112.92 \\
SL 135 & 116.22 & SL 141 & 113.89 \\
\hline CS - & 154 & Chin & \\
\hline
\end{tabular}

CS - Chinese quads type 1. CL - Chinese quads type 2.

SS - SLAC Short quads. SL - SLAC Long quads. 
Table 8 The excitation table for Chinese quadi le type 1 no. 4.

\begin{tabular}{ll}
\hline Current(A) & Gradient(Gauss $/ \mathrm{cm})$ \\
\hline 3.5 & 82.60195 \\
7.0 & 161.4780 \\
10.5 & 239.81675 \\
14.0 & 318.04635 \\
17.5 & 395.930 \\
21.0 & 474.452 \\
24.5 & 552.5505 \\
\hline
\end{tabular}

${ }^{1}$ The magnet is excited to full power, then brought down to

zero, the excitation was obtained by gradually increase the current.

2 The best fit is: $G=2.747388+22.4785351$.

${ }^{3}$ The effective length is $10.00 \mathrm{~cm}$.

Table 9 The excitation table for Chinese quadrupole type 2 no. 3.

\begin{tabular}{ll}
\hline Current(A) & Gradient(Gauss/cm) \\
\hline 3.5 & 79.22285
\end{tabular}

$\begin{array}{ll}7.0 & 159.327575\end{array}$

$\begin{array}{ll}10.5 & 238.0729\end{array}$

$14.0 \quad 317.6825$

$17.5 \quad 395.99225$

$21.0 \quad 475.09525$

$24.5 \quad 553.581$

${ }^{3}$ The magnet is excited to full power, then brought down to

zero, the excitation was obtained by gradually increase the current.

2 The best fit is: $G=1.092168+22.570705 I$.

3 The effective length is $19.99 \mathrm{~cm}$. 
Table 10 The excitation table for short SLAC quadrupole 153.

\begin{tabular}{ll} 
Current(A) & Gradient(Gauss $/ \mathrm{cm})$ \\
\hline 0.7 & 136.3408 \\
1.4 & 252.5708 \\
2.1 & 377.4048 \\
2.8 & 493.424 \\
3.5 & 612.8535 \\
4.2 & 731.4085 \\
4.9 & 849.028 \\
5.6 & 964.5245 \\
6.3 & 1080.4165 \\
7.0 & 1186.6385
\end{tabular}

IThe magnet is excited to full power, then brought down to zero, the excitation was obtained by gradually increase the current.

2 The best fit is: $G=22.842167+167.6932 I$.

${ }^{3}$ The effective length is $10.53 \mathrm{~cm}$. 
Table 11 The excitrtion table for long SLAC quadrupole 135

\begin{tabular}{ll} 
Current(A) & Gradient(Gauss $/ \mathrm{cm})$ \\
\hline 0.7 & 141.34233 \\
1.4 & 257.78066 \\
2.1 & 377.929333 \\
2.8 & 499.5900 \\
3.5 & 621.3633 \\
4.2 & 743.160667 \\
4.9 & 866.03733 \\
5.6 & 988.611667 \\
6.3 & 1109.4165 \\
7.0 & 1226.52866 \\
\hline
\end{tabular}

${ }^{1}$ The magnet is excited to full power, then brought down to zero, the excitation was obtained by gradually increase the current.

2 The best fit is: $G=16.4289+173.179357 I$.

${ }^{3}$ The effective length is $20.95 \mathrm{~cm}$. 
Table 12 The list of linear excitation relation.

\begin{tabular}{|c|c|c|c|}
\hline Mag. Type & $\int G d z($ Gauss $/ \mathrm{cm} \cdot \mathrm{cm})$ & Mag. T & e $\int G d z(G a u s s / c m \cdot c m)$ \\
\hline CS 1 & $27.5578+225.4718 \mathrm{I}$ & CS 2 & $27.5914+225.7463 \mathrm{I}$ \\
\hline CS 3 & $27.4739+224.7854 I$ & CS 4 & $27.4739+224.7854 I$ \\
\hline CS 5 & $27.5242+225.1972 I$ & CS 6 & $27.5326+225.2659 \mathrm{I}$ \\
\hline CS 7 & $27.6677+224.1677 \mathrm{I}$ & CS 8 & $27.4991+224.99131$ \\
\hline CS 9 & $27.3900+224.0990 I$ & CS 10 & $27.5075+225.0599 \mathrm{I}$ \\
\hline$\overline{C L} 1$ & $21.8493+451.5362 \mathrm{I}$ & CL 2 & $21.7618+449.7278 \mathrm{I}$ \\
\hline CL 3 & $21.8325+451.1884 I$ & $\mathrm{CL} 4$ & $21.7955+450.4233 \mathrm{I}$ \\
\hline$\overline{S S 179}$ & $239.1322+1755.5623 I$ & SS 157 & $239.3747+1757.06926 \mathrm{I}$ \\
\hline SS 158 & $237.07957+1740.4931 I$ & SS 163 & $240.3227+1764.3025 I$ \\
\hline SS 182 & $237.6953+1745.0139 I$ & SS 2.31 & $236.9154+1739.2876 I$ \\
\hline SS 218 & $236.9154+1739.2876 \mathrm{I}$ & SS 201 & $234.6164+1722.4101 I$ \\
\hline SS 242 & $240.3638+1764.6039 I$ & SS 155 & $241.1848+1770.6315 I$ \\
\hline SS 154 & $242.9912+1783.8924 I$ & SS 224 & $238.2701+1749.2333 I$ \\
\hline SS 153 & $240.5280+1765.8094 \mathrm{I}$ & SS 204 & $242.2522+1778.4675 \mathrm{I}$ \\
\hline$\overline{\text { SL } 104}$ & $334.6142+3527.21511$ & SL 119 & $339.0942+3574.4394 \mathrm{I}$ \\
\hline SL 125 & $339.4773+3578.4782 I$ & SL 130 & $332.8163+3508.2633 I$ \\
\hline SL 135 & $342.5426+3610.78961$ & SL 141 & $335.6752+3538.3998 \mathrm{I}$ \\
\hline
\end{tabular}

The current I unit is Ampere.

CS - Chinese quads type 1 , the effective length is $10.00 \mathrm{~cm}$.

CL - Chinese quads type 2, the effective length is $19.99 \mathrm{~cm}$.

SS - SLAC short quads, the effective length is $10.53 \mathrm{~cm}$.

SL - SLAC long quads, the effective length is $20.85 \mathrm{~cm}$. 


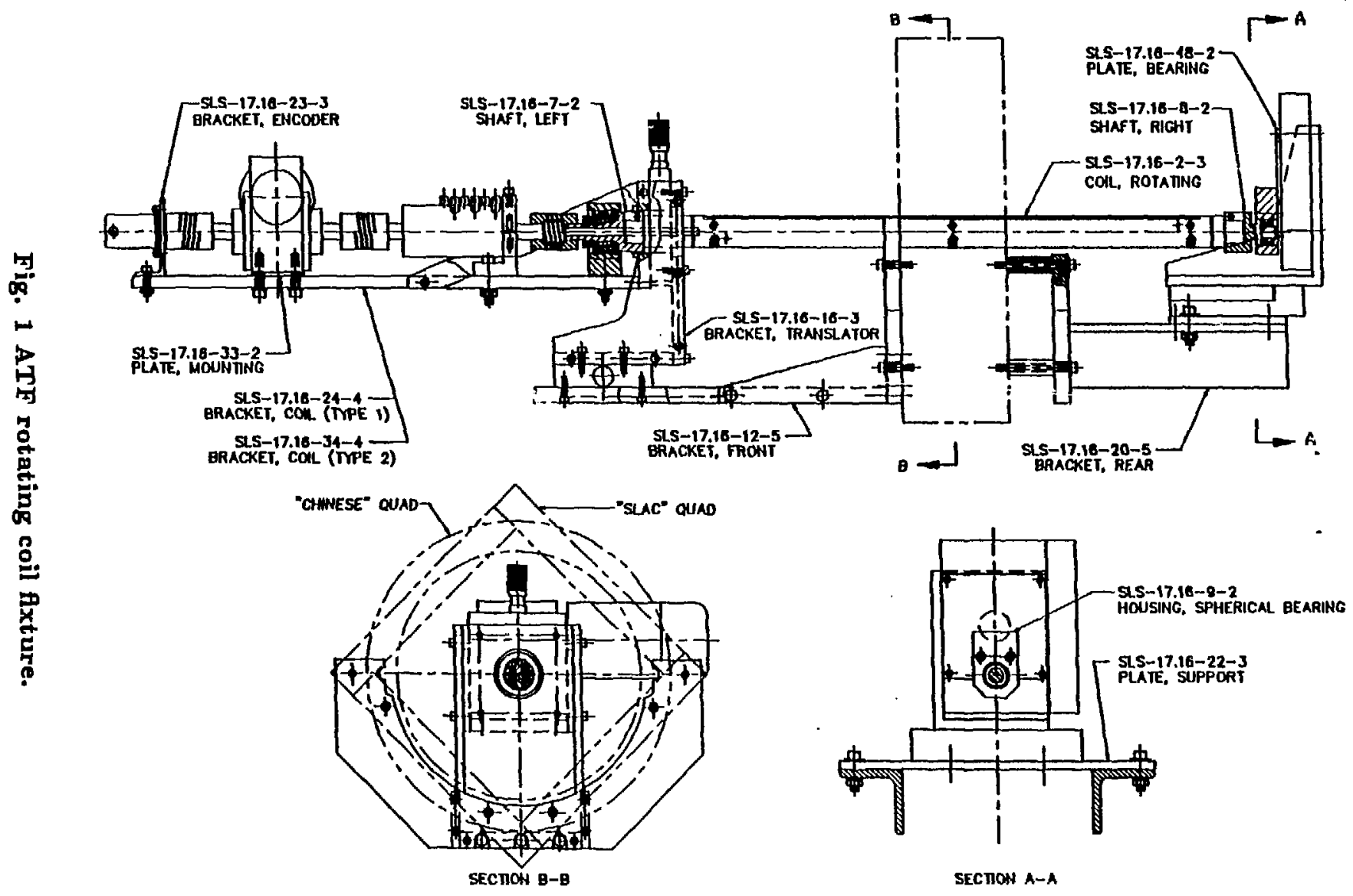




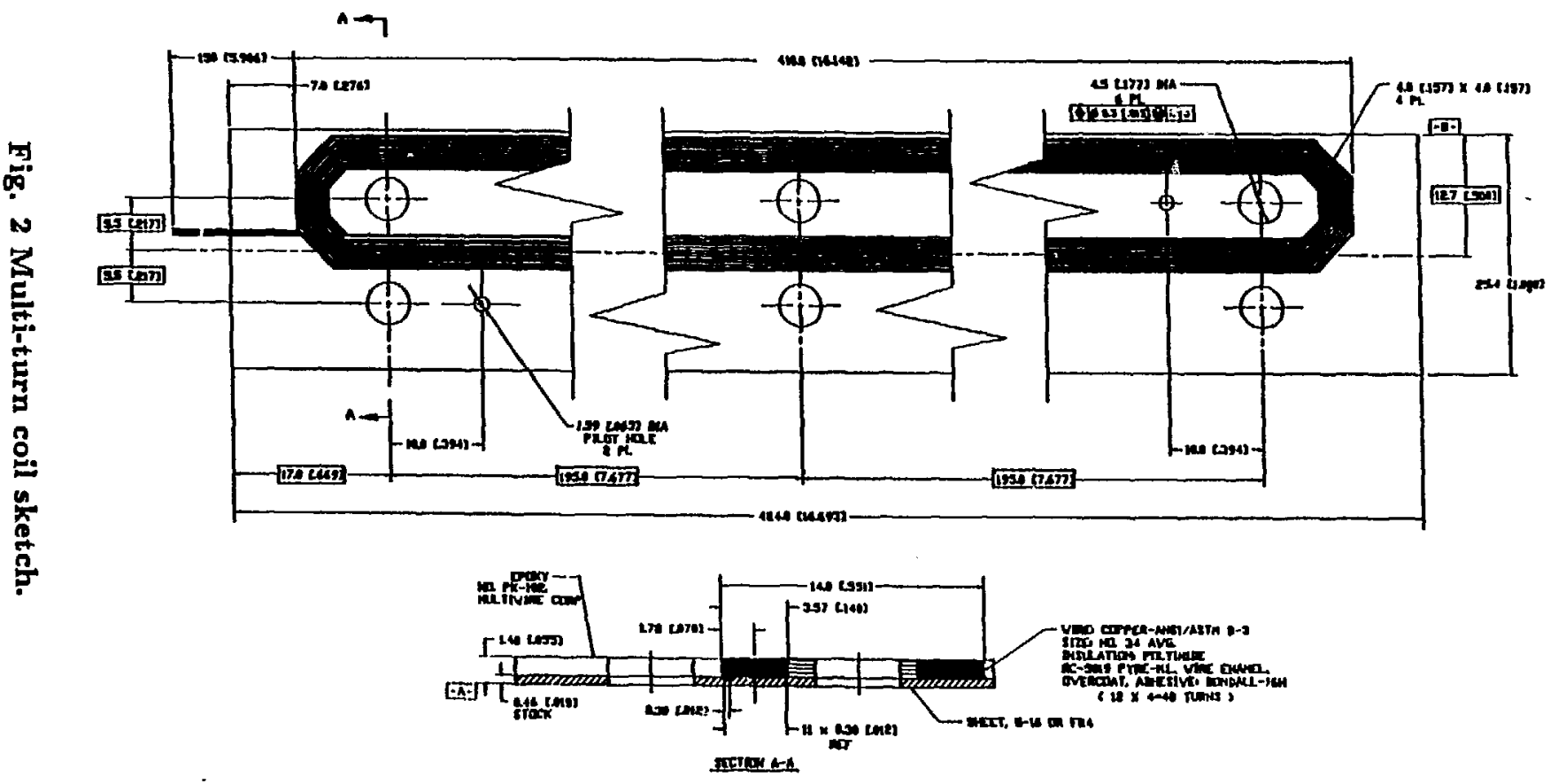




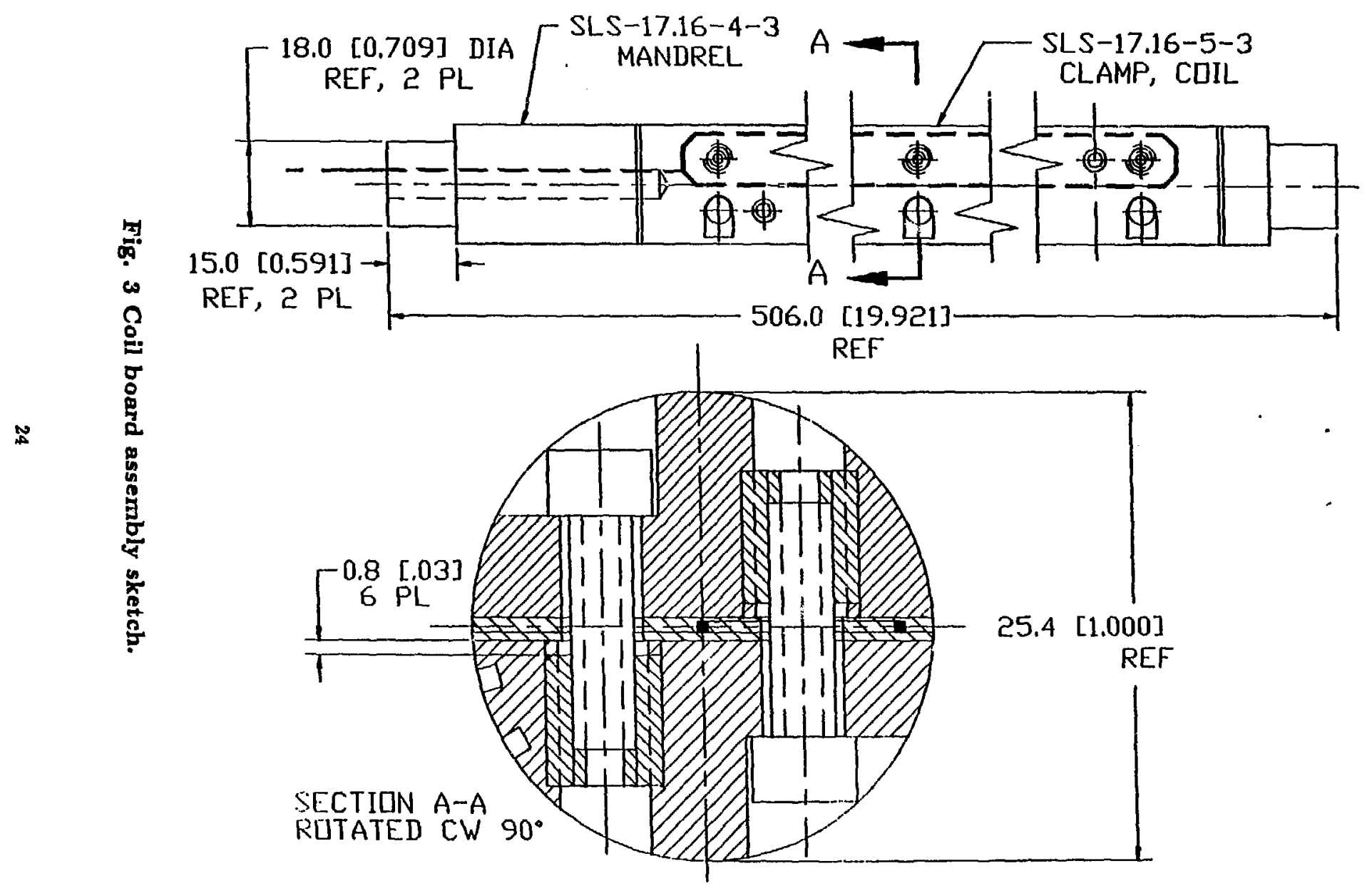




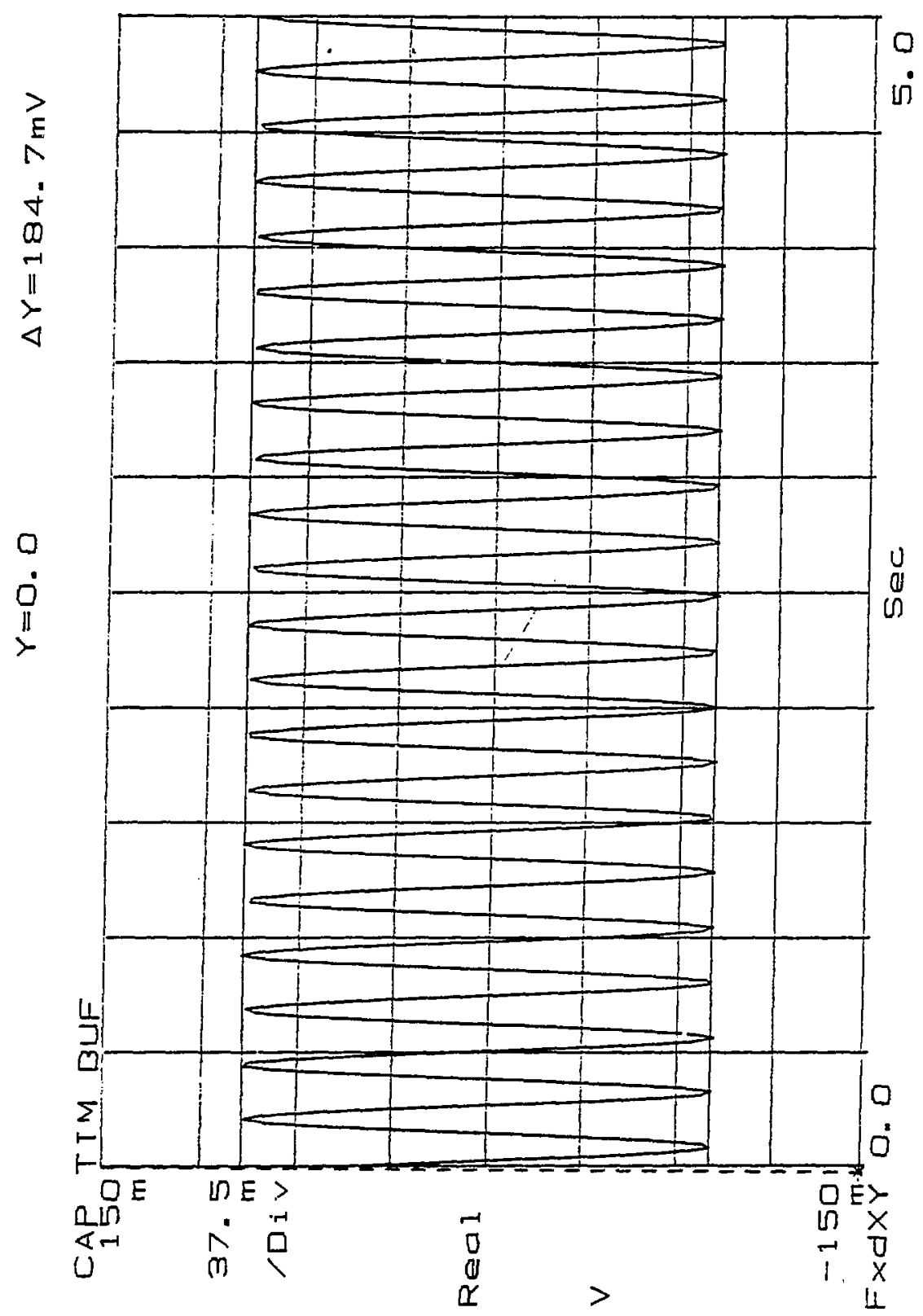

Fig. 4 Time varied voltage induced by the quadrupole. 


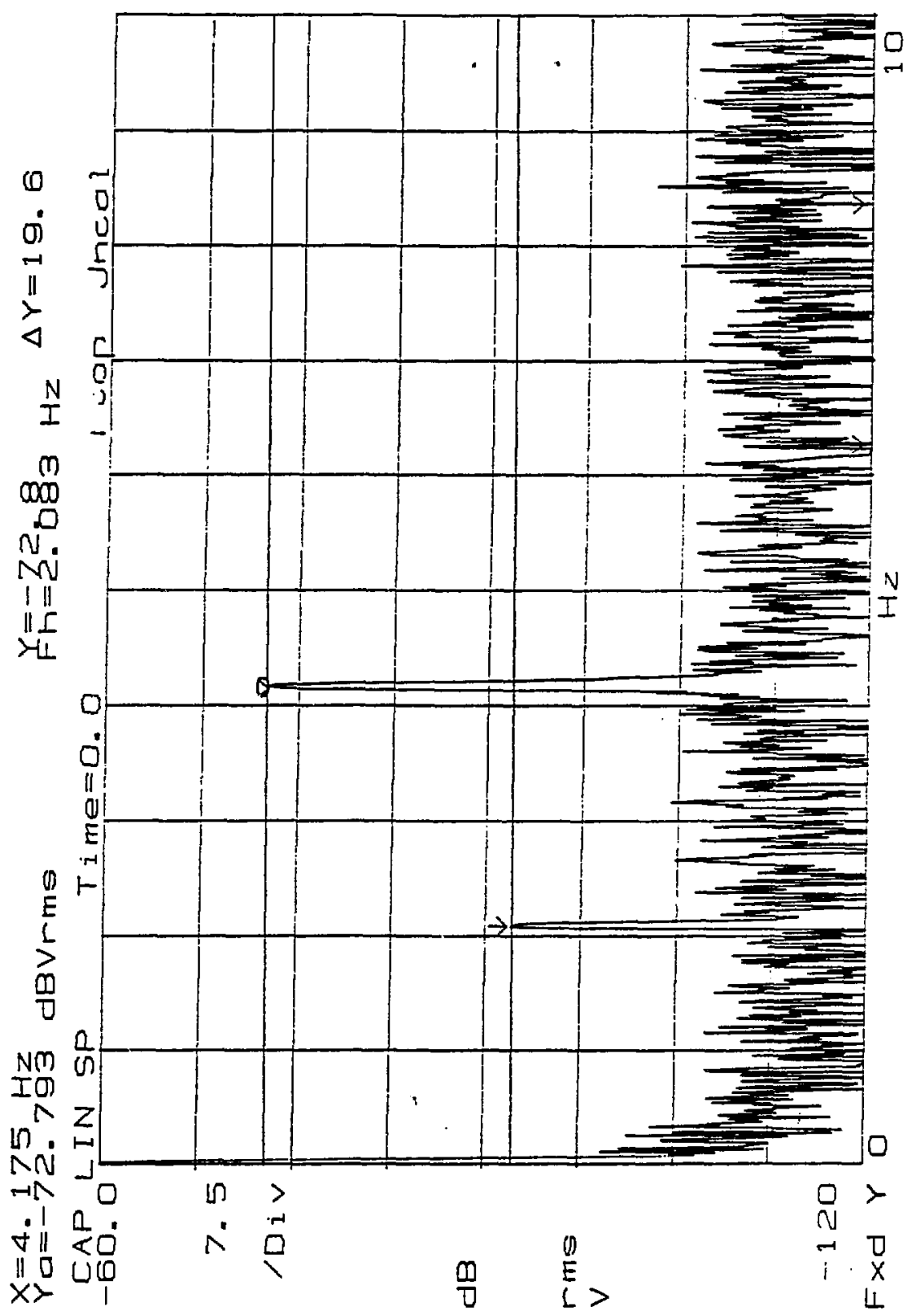

Fig. 5 The power spectrum of the quadrupole before the magnet was powered. 


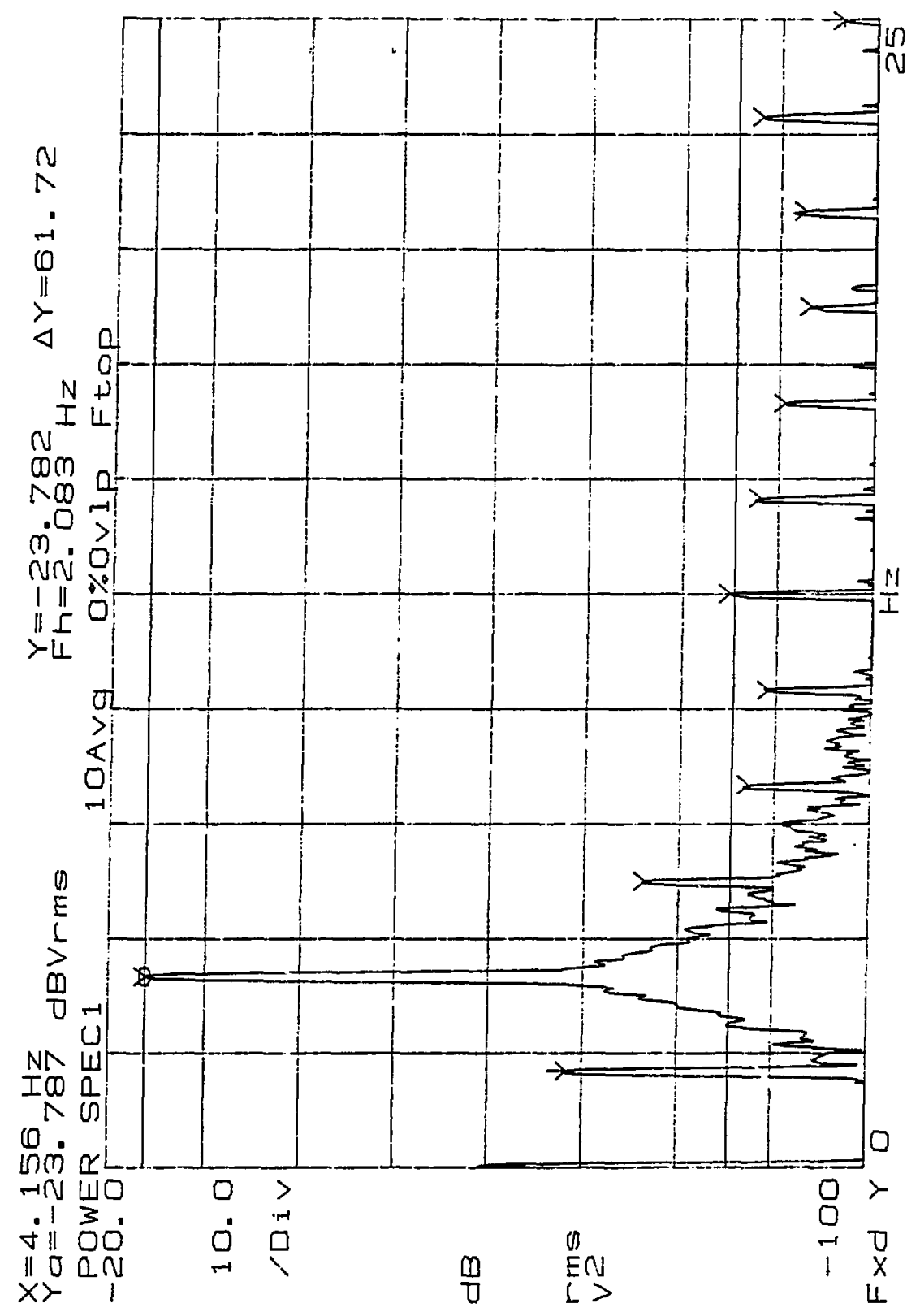

Fig. 6 The power spectrum of the quadrupole at the mechanical center. 


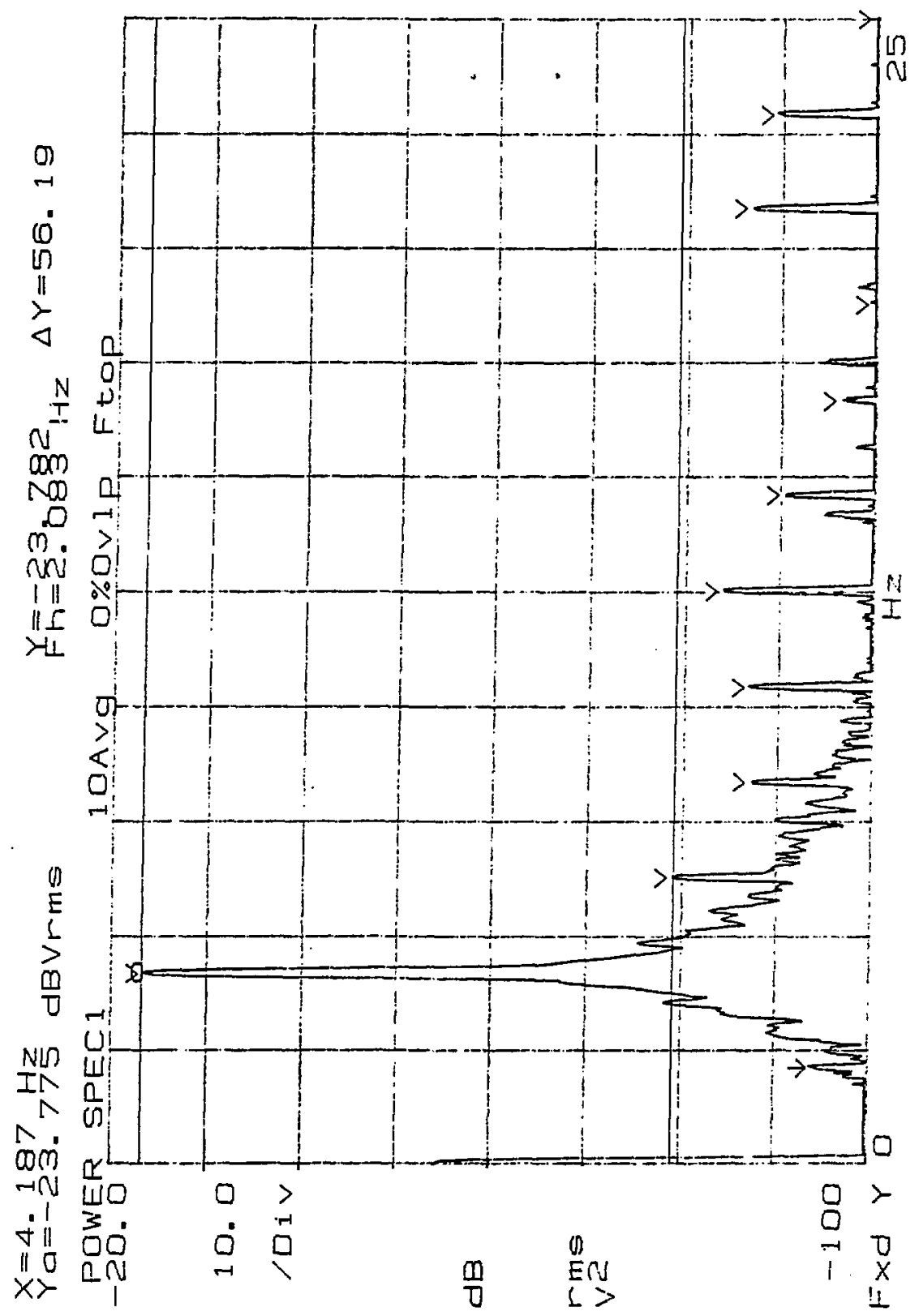

Fig. 7 The power spectum of the quadrupole at the magnetic center. 


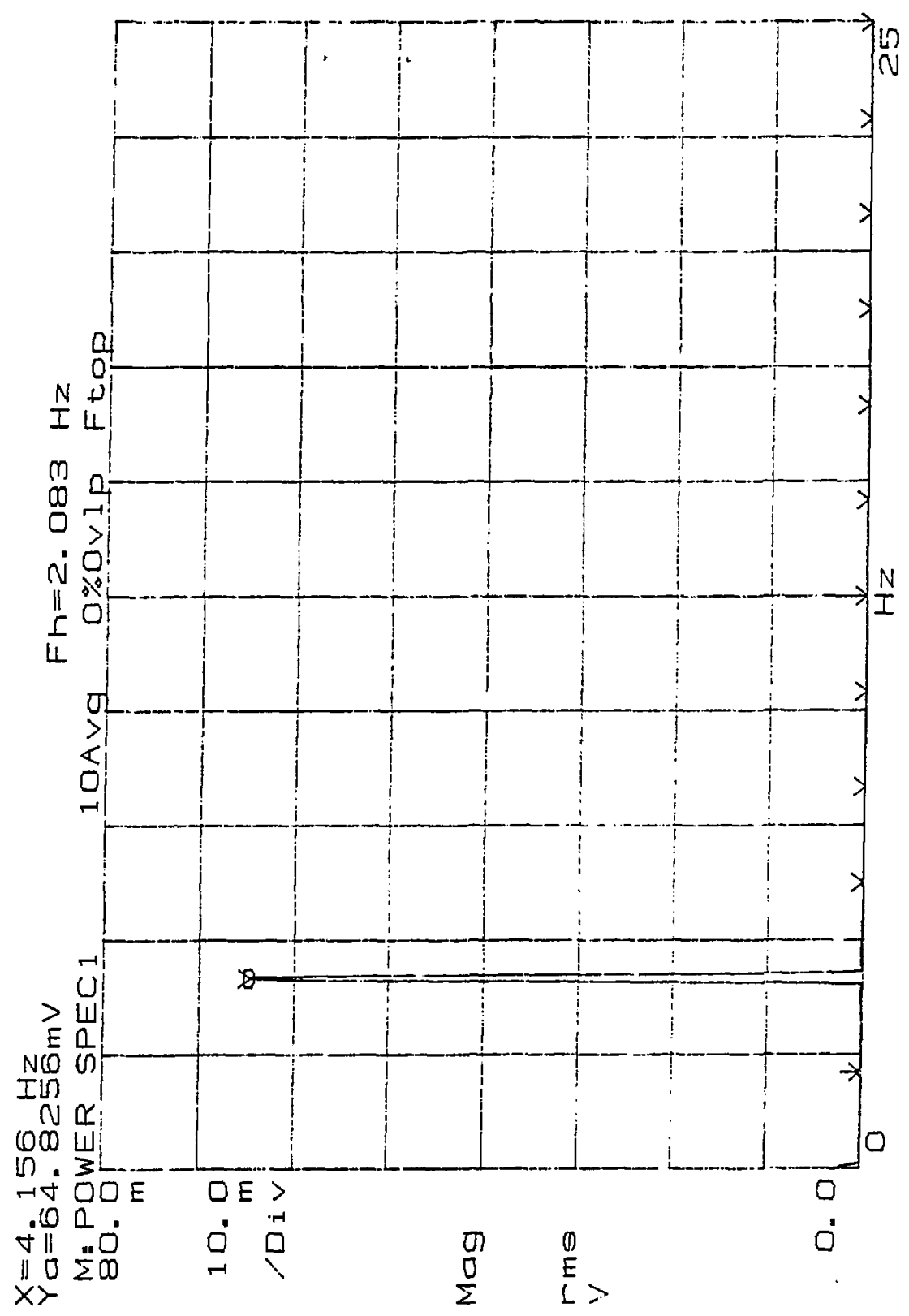

Fig. 8 RMS voltage induced on the coil by each harmonic. 
(G)

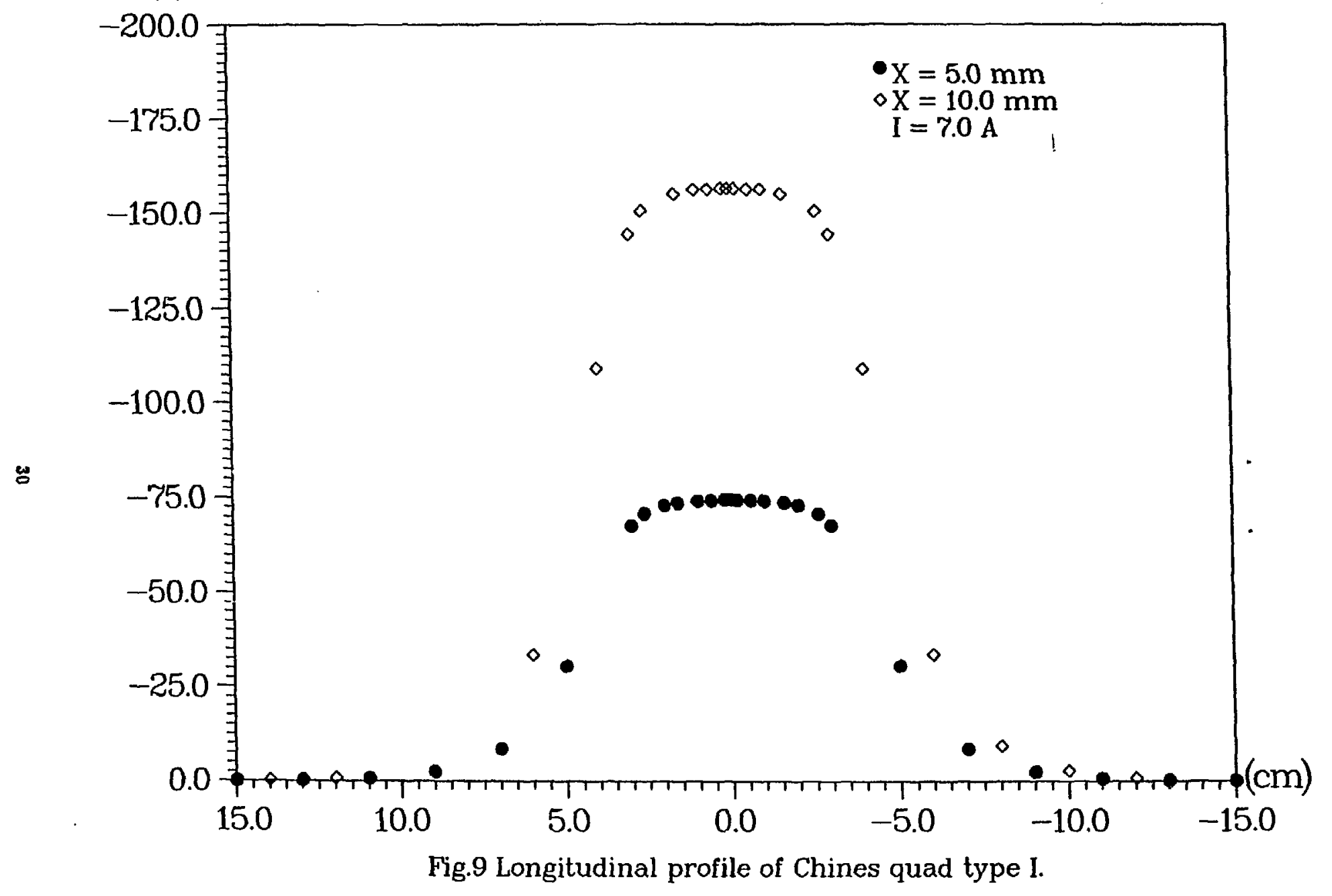


(G)

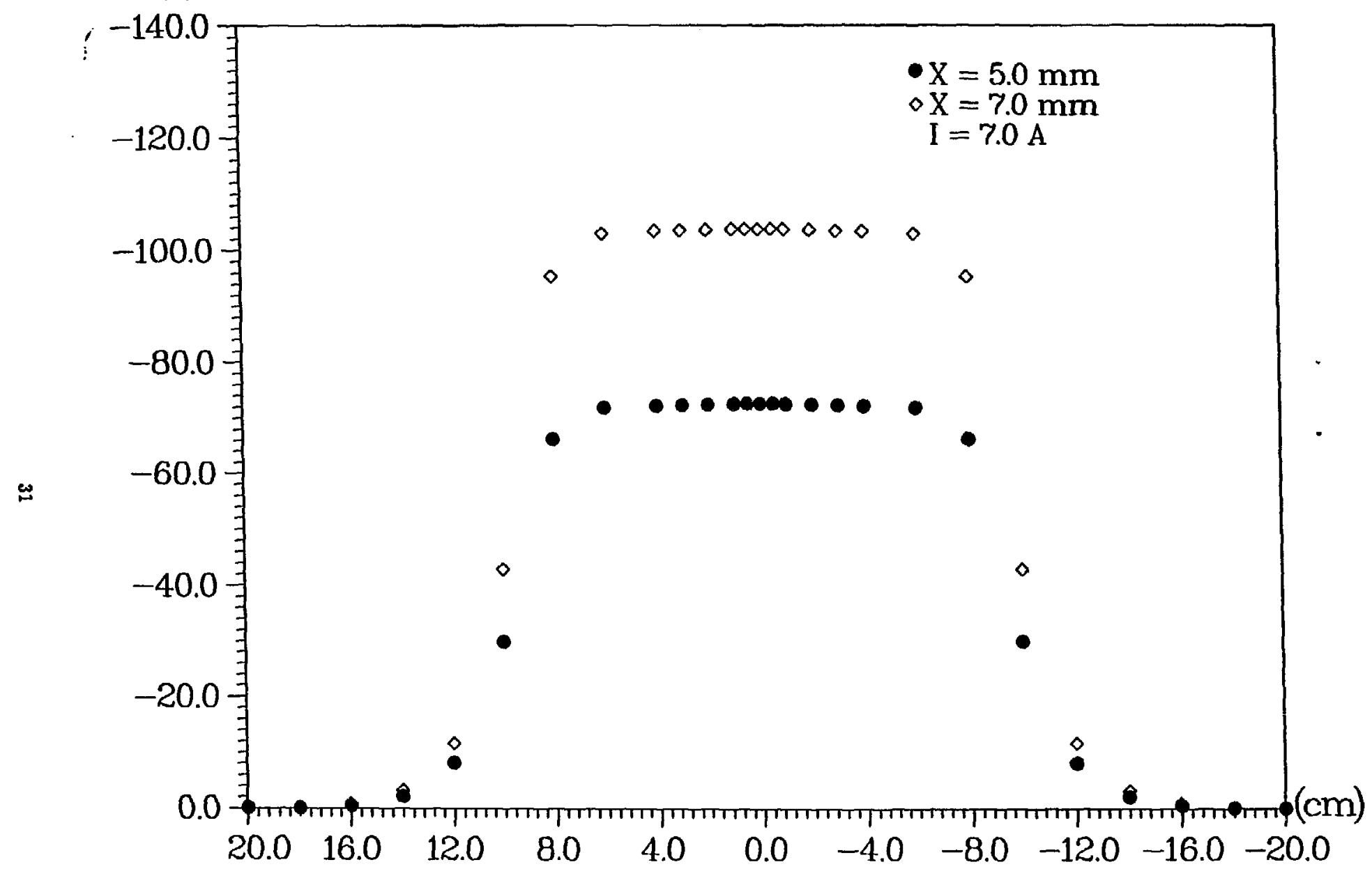

Fig.10 Longitudinal profile of Chinese quad type II. 


\section{(G)}

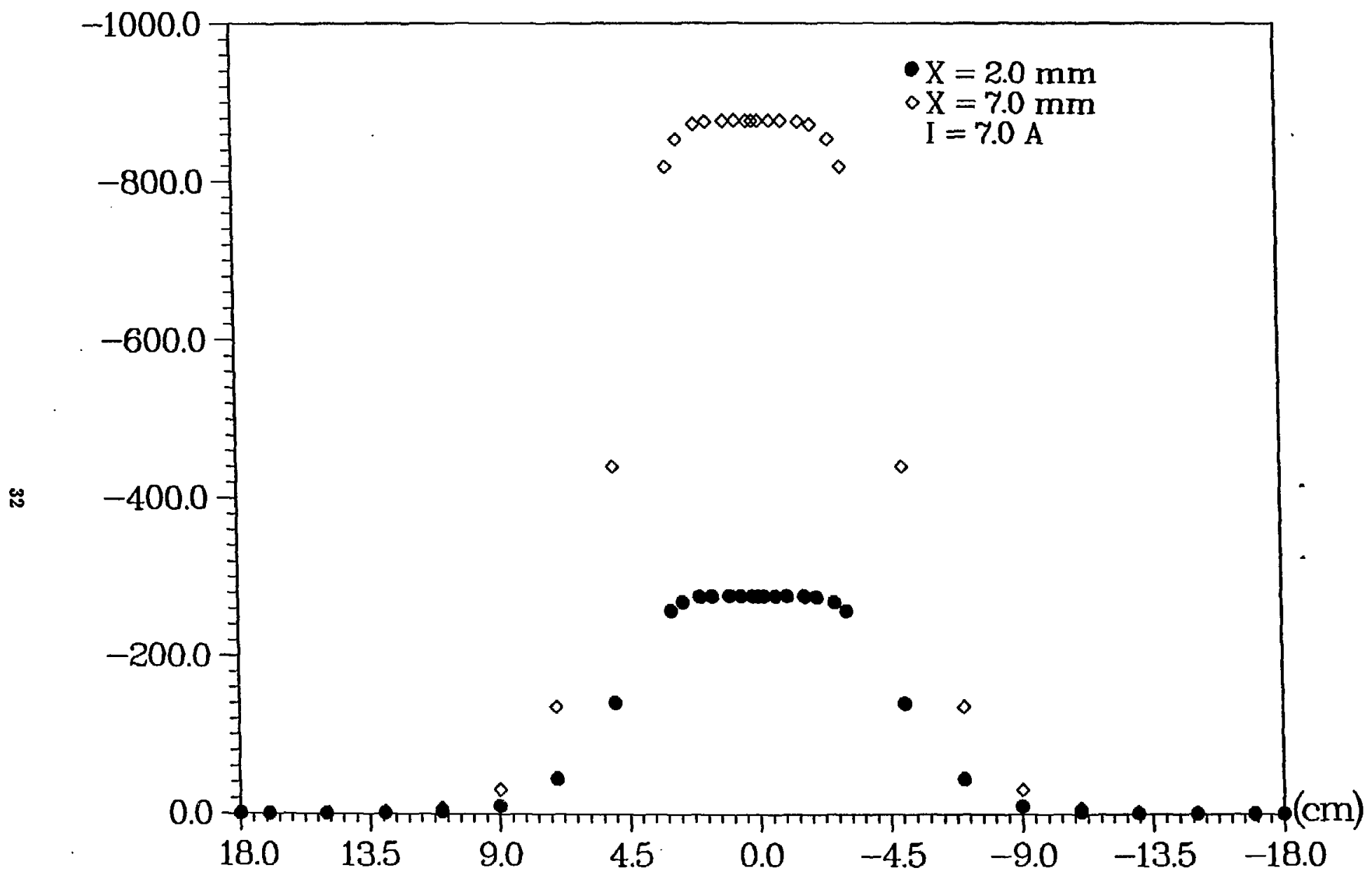

Fig.11 Longitudinal profile of SLAC short quadrupole. 
(G)

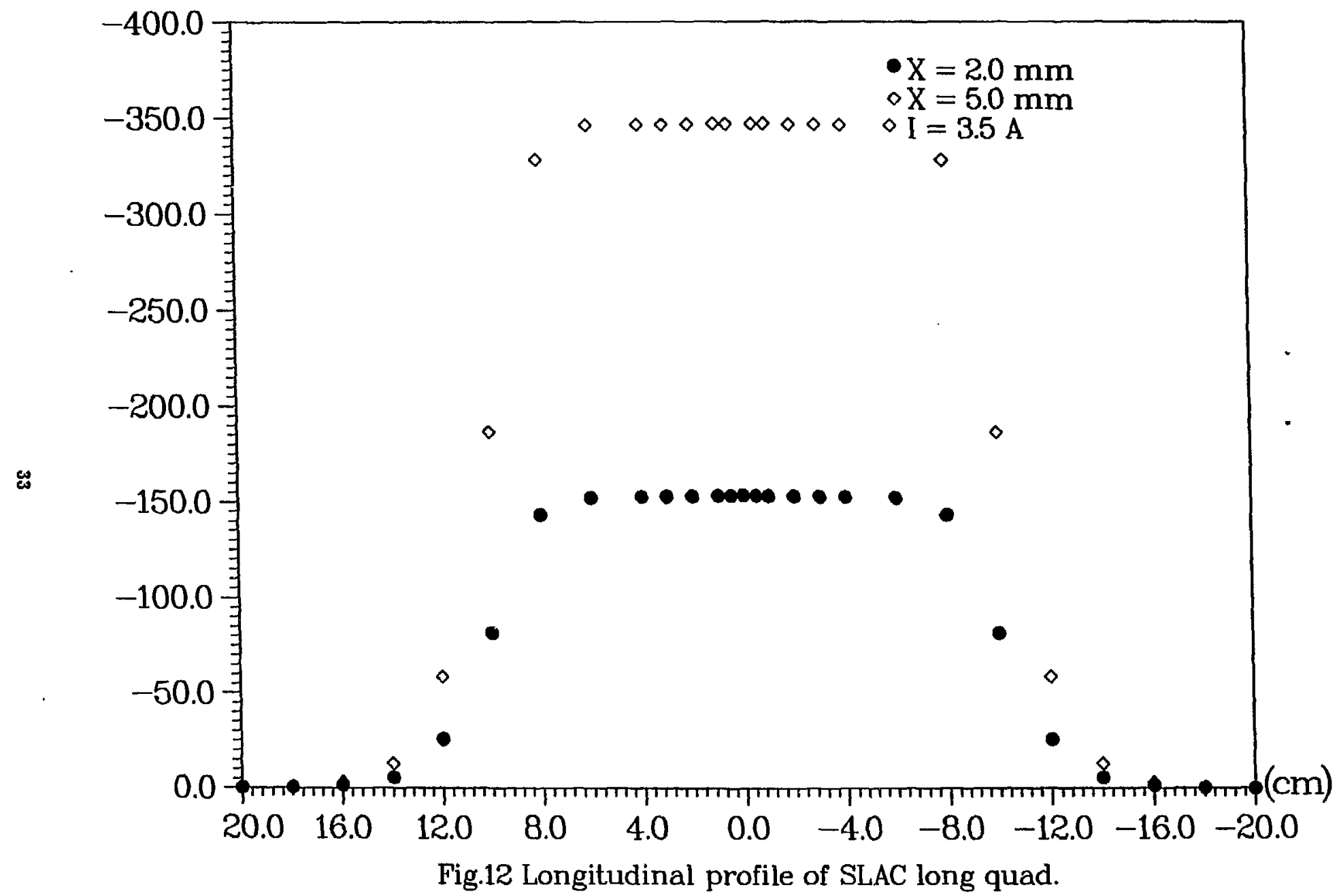

